\title{
A INSUFICIÊNCIA EDUCACIONAL NA TRANSIÇÃO SECUNDÁRIO- UNIVERSIDADE NOS TEMPOS DA COVID-19 COMO REFERENCIAL PARA A FORMAÇÃO DE PROFESSORES
}

\author{
José Manuel Bautista Vallejo ${ }^{1}$ \\ https://orcid.org/0000-0002-4238-9507 \\ Rafael Manuel Hernández-Carrera ${ }^{2}$ \\ https://orcid.org/0000-0002-7722-8105 \\ Cintia Almeida da Silva Santos ${ }^{3}$ \\ https://orcid.org/0000-0002-1832-3751
}

Resumo: Este artigo oferece uma revisão documental sobre a chamada transição do Ensino Médio para a Universidade, ou seja, a passagem do Ensino Médio para a Universidade. Propõe a análise das dificuldades que os alunos têm no primeiro ano do ensino universitário, sendo a continuação daquelas semelhantes que os alunos têm nos últimos anos do ensino secundário. São propostas soluções direcionadas através de três linhas de trabalho: o aprimoramento da colaboração do Ensino Médio, o uso da inovação superando a ideia que temos sobre ela e a gestão de competências nos processos de ensino-aprendizagem de uma forma real e eficaz, tudo no âmbito do fortalecimento da formação docente, o que ficou evidenciado na atual crise provocada pelo COVID-19.

Palavras-chave: secundário; faculdade; transição; inovação; competências.

\footnotetext{
1 Professor Doutor da Faculdade de Ciências da Educação da Universidade de Huelva (UHU). E-mail: josembau@gmail.com.

2 Professor e Diretor do Departamento de Didática e Organização Escolar da Universidade Internacional da Rioja (UNIR). E-mail: rmhercar@upo.es.

3 Doutora em Ciência, Tecnologia e Sociedade pela UFSCar. Bibliotecária-Documentalista no Instituto Federal de Educação, Ciência e Tecnologia de São Paulo (IFSP). E-mail:

cintiasert@hotmail.com.
} 


\section{THE EDUCATIONAL FAILURE IN THE SECONDARY-UNIVERSITY TRANSITION IN TIMES OF COVID-19 AS A FRAMEWORK FOR TEACHER TRAINING}

Abstract: This article offers a documentary review of the so-called transition from Secondary to University, that is, the transition from the Secondary education to the University. It proposes the analysis of those difficulties that students have in the first year of their university studies, being the continuation of those similar to the students in the last years of Secondary. Solutions are proposed through three lines of working: the improvement of Secondary-University collaboration, the use of innovation surpassing the idea we have about it and the management of competences in the teaching-learning processes in a real and effective way, all this within the framework of strengthening teacher training, something that was evidenced in the current crisis by COVID-19.

Keywords: secondary; university; transition; innovation; competences.

\section{EL FRACASO EDUCATIVO EN LA TRANSICIÓN SECUNDARIA-UNIVERSIDAD EN TIEMPOS DE COVID-19 COMO MARCO PARA LA FORMACIÓN DEL PROFESORADO}

Resumen: Este artículo ofrece una revisión documental sobre la llamada transición Secundaria-Universidad, es decir, el paso de la etapa Secundaria a la Universidad. En él se propone el análisis de aquellas dificultades que el alumnado tiene en el primer curso de sus estudios universitarios, siendo la continuación de aquellas similares que posee el alumnado en los últimos años de la Secundaria. Se plantean soluciones encaminadas a través de tres líneas de trabajo: la mejora de la colaboración Secundaria-Universidad, el uso de la innovación superando la idea que tenemos sobre la misma y el manejo de las competencias en los procesos de enseñanza-aprendizaje de una forma real y efectiva, todo ello en el marco del fortalecimiento de la formación del profesorado, algo que se evidenció en la actual crisis por la COVID-19.

Palabras clave: secundaria; universidad; transición; innovación; competencias. 


\section{Introducción}

Las últimas décadas se han caracterizado por una creciente preocupación por el sistema educativo en el marco de una inquietud por la mejora de la calidad, la pertinencia y la equidad. También por una preocupación por la utilidad de los conocimientos que son enseñados y aprendidos. Otro marco que se ha sostenido, un poco más concreto, es el de elevar la competencia de la Educación Superior, en ambientes cada vez más competitivos en donde no es fácil subsistir (MORA, 2018).

En contextos como el europeo, a nivel académico y, también, político, las declaraciones y comunicados de La Sorbona, Bolonia, Salamanca, Praga o Berlín insistieron en la conveniencia de crear un Espacio Europeo de Educación Superior (EEES), armonizando, pero respetando las peculiaridades de cada uno (BAUTISTA VALLEJO, 2005). Estas medidas han inspirado, sin duda, muchas de las decisiones que se han tomado en el llamado MERCOSUR educativo (KROTSCH, 1997; CICARÉ, 2019).

Todos los informes y reformas durante estos años han incidido en un denominador común: la necesidad de que los sistemas se adapten a las nuevas realidades demográfica, social, cultural, económica y jurídica y de las tecnologías de la información y comunicación (BARNETT, 2019).

La reciente crisis relacionada con la enfermedad COVID-19, provocada por el coronavirus SARS-CoV-19, puso de manifiesto la necesidad de establecer estrategias eficaces desde el punto de vista de los procesos de enseñanza-aprendizaje para afrontar el cierre de los centros educativos, a través de, entre otras cosas, una enseñanza online distribuida y útil para toda la comunidad educativa.

Se asiste, de esta forma, al advenimiento de nuevas transformaciones. Toda la enseñanza, universitaria y no universitaria, está evidenciando el mayor experimento en décadas, con la importancia de que es un experimento a lo largo y ancho de todo el mundo: su transformación en un sistema de enseñanza virtual, mixto o al completo, como consecuencia del impacto de la pandemia por COVID-19.

En este marco, nuevos objetivos están siendo planteados a toda marcha, en un intento que va desde la supervivencia de una parte del sistema a la transformación del mismo para adaptarse a la nueva situación (CORNOCK, 2020) que, de momento, se presenta con grandes incógnitas en relación a su proceso y, también a sus resultados. 
En medio de todas estas pretensiones y atisbando el futuro, la situación actual y los objetivos propuestos solo pueden alcanzarse incidiendo en determinados problemas que en las últimas décadas no han quedado resueltos en la mayoría de las universidades: el problema del rendimiento y los factores que influyen en el mismo, el de la calidad, el del fracaso educativo, el de la igualdad de oportunidades y equidad, etc. A esto se añade, hoy de forma extraordinaria, el interés por aumentar la capacidad investigadora de estas instituciones de Educación Superior (CASTILLO; MORÉ, 2018).

Como consecuencia de ello, la discusión sobre muchos de estos elementos se ha prolongado por más de 20 años (BAUTISTA-VALLEJO; ESPIGARES-PINAZO; HERNÁNDEZ-CARRERA, 2019). La misma no ha obviado la parte de responsabilidad de la etapa inmediatamente anterior, la Educación Secundaria, la cual se ha visto igualmente afectada por todos estos problemas, admitiendo, al igual que la universidad, el reto de una formación virtual tratando de reducir las distancias (SOUSA; SANTOS JUNIOR, 2019; TORI, 2017).

Si se considera a la etapa Secundaria no simplemente como un período anterior a la etapa universitaria, sino una etapa clave para el éxito en la universidad y, también, para la vida, el problema de la naturaleza de estos dos espacios, sus relaciones y, en concreto, la transición entre ambas cobra, sin duda, mucha importancia.

El objetivo de este trabajo es el análisis, a través de una revisión documental, de los principales problemas que afectan a la transición Educación SecundariaUniversidad, problemas que comienzan en la etapa Secundaria y que se arrastran de forma casi idéntica a los niveles superiores, en concreto la Universidad, todo ello en el marco del fortalecimiento de la formación del profesorado, algo que se evidenció en la actual crisis por la COVID-19.

En cuanto a la metodología empleada, se ha llevado a cabo una revisión documental descriptiva (ISERN; CANELA-SOLER, 1994; KAUARK; MANHÃES; MEDEIROS, 2010) de una serie de documentos seleccionados entre múltiples fuentes consultadas, en bases de datos y buscadores especializados, fundamentalmente Google Académico, Web of Science (WOS) y Dialnet.

Las mismas permitieron el acceso a bases consistentes y confiables y el manejo y análisis de un gran volumen de publicaciones científicas a través de una serie de 
categorías, que tienen las características de ser significativas, claras, excluyentes y replicables (BARDIN, 1996), en una espiral auto-reflexiva continua (HERNÁNDEZ CARRERA, 2014).

Las categorías usadas fueron: Espacio Europeo de Educación Superior, modelo de enseñanza y aprendizaje por competencias, transición Secundaria - Universidad, rendimiento académico, fracaso escolar, abandono escolar. Se manejaron más de 200 documentos de entre los que fueron seleccionados y analizados 59.

\section{Elección del modelo por competencias y la reforma universitaria}

Para entender una parte de estos problemas existentes es necesario remontarse a las recientes reformas del sistema educativo que afectaron tanto a lo universitario como a las etapas no universitarias. En concreto, la reforma de la Educación Superior Europea (Espacio Europeo de Educación Superior-EEES) afectó tanto a la universidad como a la educación obligatoria, por cuanto que desde un punto de vista pedagógico era necesario plantear los mismos criterios (GONZÁLEZ; WAGENAAR, 2003).

Desde finales de los años 90 del siglo pasado, un amplio conjunto de países occidentales apostó y desarrolló en sus respectivos sistemas educativos, universitarios y no universitarios, el denominado modelo de enseñanza-aprendizaje basado en competencias.

Uno de los proyectos que impulsaron de forma más directa estas reformas, sobre toda la universitaria, fue el Proyecto Tuning (Tuning Educational Structures in Europe, Armonización de las Estructuras Educativas en Europa), proyecto dirigido por González y Wagenaar (2003). En el marco del mismo se llevó a cabo un análisis conceptual, es decir, un análisis de los términos clave para entender la reforma en ciernes, la cual consideraba que algunos de éstos, como capacidad, atributo, habilidad, destreza, competencia, etc., tenían cierto grado de coincidencia en los significados, pero que en ocasiones eran usados de forma imprecisa.

Para González y Wagennar (2003), la elección del término competencias está motivada por algunas de estas ideas iniciales, fundamentalmente el hecho de que se presenta como un concepto aglutinador de significados diversos, todos los cuales transmiten lo que la persona es capaz de ejecutar, el grado de preparación, suficiencia 
o responsabilidad para ciertas tareas (DUQUE; CONTRERAS; GARCÍA, 2019; PRIETO, 2008).

Fruto de este análisis, el concepto de competencias se presenta con un enfoque integrador. Para Heywood, Gonczi y Hager (1993), el mismo representaba una dinámica combinación de atributos, algo que se ha mantenido en el tiempo (MARÍN, 2018). Una de las virtudes que posee el término competencias es que como parte de un proceso educativo podemos alcanzar un determinado desempeño competente (ARGUDÍN, 2000), lo cual conecta con la labor realizada en las instituciones universitarias. Las competencias tienen que ver con conocer y comprender, saber cómo actuar y saber cómo ser, algo que recuerda el camino abierto por el documento de Delors (1996).

Buena parte de la reflexión sobre las competencias se refiere a su naturaleza pedagógica, advirtiendo los críticos que el trabajo pedagógico no se reduce solo a la acumulación de saber-hacer y a la práctica de ejercicios mecánicos. También se refiere a la capacidad de crear situaciones generadoras de sentido que se encaminen hacia el descubrimiento y al aporte original, esto es, el trabajo pedagógico se refiere también a la capacidad de pensar (MEIRIEU; MONTENEGRO GÓMEZ, 2019).

Los atributos mencionados se establecen con relación al conocimiento y sus aplicaciones, es decir, las aptitudes, destrezas y responsabilidades, las cuales describen el grado de aptitud con que una persona es capaz de desempeñarlos (ÁLVAREZ COLÍN, 2000). Esta es la razón por la que, además, se habla del desempeño y su relación con las competencias (TORRES-LÓPEZ et al., 2018).

Años antes (2001, obra original de 1994), se lleva a cabo un análisis de las competencias asumiendo dos formas de racionalidad o ideologías en torno a éstas: la ideología de la competencia operacional y la ideología de la competencia académica (BARNETT, 2001).

Así, afirma que,

[...] al desarrollar el análisis de este libro, nos hemos centrado en un conjunto de términos, que incluye competencia, resultados, habilidades, transferibilidad, empresa y acumulación de créditos, que juntos constituyen una definición operacional de competencia. Esta concepción operacional de la competencia es [...] una ideología en el sentido estricto del término. Esto quiere decir que adquiere poder ofreciendo definiciones de la realidad que al mismo tiempo 
alimentan un conjunto de intereses sociales. La percepción, la parcialidad y el programa se desarrollan al mismo tiempo (BARNETT, 2001, p. 262).

Lo problemático de este enfoque, hegemónico en la actualidad, pero sin la fuerza suficiente como para involucrar de manera profunda a toda la comunidad educativa (BAUTISTA VALLEJO; LÓPEZ JARA, 2019; ROLÓN, 2016) es que apuesta, usando la terminología de Barnett (2001), por la definición operacional de competencia. Hoy se da una relación entre una determinada forma de racionalidad e ideología dominante y la puja de un poder político determinado en connivencia con un poder económico. Esto ha influido de manera decisiva en la manera de entender la educación. Son más usados los términos competencia, empresa y transferibilidad que los de objetividad, verdad y disciplinas (BARNETT, 2001). El dominio de este vocabulario es total y cuenta con respaldos de todo tipo, incluso los poderes del Estado, así la dificultad de plantear un lenguaje diferente.

Esta forma de lenguaje, expresado en las reformas educativas (BARNETT, 2019), es una forma de ejercer el poder y establecer marcos (PEREIRA; PASSOS, 2018) y, también, restricciones sobre lo que es el ser humano ideal: se enmarca en una concepción de lo que es el ser humano y lo que, de seguir esta lógica, va a llegar a ser.

La revisión documental pone de manifiesto que el empleo de los niveles más altos de cognición (las metacompetencias y la metacognición) en las instituciones educativas puede ser considerado como un síntoma de la búsqueda de nuevas definiciones de razón (PERRY; LUNDIE; GOLDER, 2018; SAFITRI et al., 2018), lo que permitiría al alumnado un conocimiento más profundo de su yo y de los procesos formativos que le afectan.

\section{La transición Secundaria - Universidad}

Para Rodríguez Espinar (2003), la revisión de la investigación puso de manifiesto un desenfoque en el estudio de la relación entre la Secundaria y la universidad. Advierte que en el análisis de la transición a la universidad se han hecho estudios que describen resultados académicos al final de un determinado período y 
seguimientos de cohortes específicas en referencia a centros concretos, pero no se ha producido un planteamiento comprensivo y profundo que permita constatar el conjunto de factores psicosociales relacionados con la calidad de las transiciones académicas. Este, por otro lado, es un aspecto clave. Es decir, la transición a la universidad es un proceso complejo que comporta para el estudiante cambios personales y vitales significativos, también sociales.

Hoy, los estudios se constituyen en una base para desarrollar estrategias de enseñanza-aprendizaje con las que paliar los problemas de adaptación (SOARES et al., 2018). Es decir, estudios abren la posibilidad de establecer estrategias de enseñanzaaprendizaje no incorporadas hasta el momento, de forma que en el supuesto de que el estudiante al ingreso a la universidad no posea una capacidad autorregulatoria desarrollada, cosa que no se cumple en la mayoría de los casos, estaremos en mejores condiciones de contribuir a reducir los bajos desempeños y todo lo que esto trae aparejado (GRAVINA; PRIETO, 2019), sobre todo durante los primeros años del periodo universitario.

Los problemas relacionados son muchos y variados (DOMÍNGUEZ; MEDINA; LÓPEZ, 2018; PIERELLA, 2018a). Puede comprobarse, por ejemplo, que la formación desde un punto de vista pedagógico es deficiente, con inadecuados planteamientos y procesos de enseñanza-aprendizaje, relaciones educativas y de comunicación entre centros de Secundaria y universitarios pobres o nulas, etc. (GIL-GALVÁN, 2019). A estos elementos las investigaciones recientes añaden la necesidad de articular más adecuadamente la Educación Secundaria con la universidad, establecer políticas de acceso y permanencia, repensar las condiciones de trabajo docente, establecer más adecuadamente la identidad del ciclo inicial y las particularidades de la profesión docente en el primer año universitario (PIERELLA, 2018a).

En la transición Secundaria-universidad, que abarca desde los últimos años de la Secundaria hasta los primeros de la universidad, el abandono en algún momento de este proceso es un tema destacado. El mismo puede contemplarse de manera general incluyendo distintas situaciones. Este abandono ofrece una variedad de matices. Yorke (1999) lo consideraba como "la no finalización" (non-completion), lo cual esconde un conjunto de situaciones diversas. Se contempla bajo esta categoría al alumnado expulsado, el abandono de estudios universitarios como una suspensión 
transitoria, el traslado de una universidad a otra, el abandono de carrera y universidad inicial, el abandono total, etc. Cada una de estas situaciones requiere en su tratamiento enfoques diferenciados. La pregunta, de momento pobremente respondida (PIERELLA, 2018b), es: ¿cuántas de ellas tienen su origen en la Secundaria?

Rodríguez, Dapía y López-Castedo (2014) afirman que la preocupación por los resultados es creciente, al mismo tiempo que la situación de estrés que están dejando las formas de evaluación y la metodología de enseñanza-aprendizaje tradicional, algo que también va aparejado a un desinterés generalizado por los procesos. Por lo general, esto lleva a las administraciones educativas y a muchas instituciones a poner interés en el rendimiento y la mejora de las competencias.

Sin embargo, en algunos países, como el caso de España, desde hace más de 30 años la tasa de abandono se sitúa en unos promedios muy altos y plantea una difícil solución (GIL-GALVÁN, 2019). Problemas similares pueden reportarse en el contexto latinoamericano (COSTA MOROSINI et al., 2012; TORRES NAVARRO, 2019). Por lo general, la reprobación de una o más asignaturas afecta a la mitad del alumnado durante su primer año de carrera y un porcentaje muy significativo se retira del sistema antes del número de años previsto en las titulaciones.

Por su parte, Smulders Chaparro (2018) afirma que el éxito o el fracaso de un estudiante, finalmente, es consecuencia de variados factores y que éstos se van acumulando desde muy temprana edad en el estudiante y su entorno, los cuales se manifiestan a lo largo su inserción académica y, de una manera especial, en la transición Secundaria-universidad.

Esta transición, entendida como proceso, presenta a lo largo del año 2020 un punto de inflexión. El establecimiento de estados de emergencia sanitaria y alarma en muchos países del mundo como consecuencia de la pandemia por COVID-19 ha generado un impacto en los sistemas educativos sin precedentes, lo cual produjo en la mayoría de los casos de forma acelerada la toma de importantes medidas educativas, siendo las más importantes las relativas a las medidas de virtualización de la enseñanza-aprendizaje (BOUZA MORA, 2020; WANG, G. et al., 2020a). En particular, las instituciones de educación superior, también las de Secundaria, trabajaron sin descanso para no detener su programación docente, para lo cual se introdujeron todo 
tipo de recomendaciones y "obligaciones" de trabajo a distancia, enseñanza virtual, etc., haciendo uso de las muchas herramientas que hasta el momento habían sido utilizadas con relativa frecuencia y eficacia.

El caso de las universidades de China, por ser el primero y afectar a millones de universitarios, ha sido muy significativo (WANG, C. et al., 2020b), al que ha seguido el de decenas de países en todo el mundo (SUND, 2020).

\section{La importancia del rendimiento académico}

El rendimiento académico, unido al problema de la tasa de abandono temprano y fracaso educativo, es, probablemente, el factor más importante para gobiernos y comunidad educativa (BANCO MUNDIAL, 2017). En este sentido, la discusión sobre las capacidades y las competencias, si bien son cuestiones vinculadas y pese a su valor teórico, no alcanzan a tener la importancia que se le da al rendimiento académico, abandono temprano y fracaso educativo (LAMAS, 2015).

Como afirma Barnett (2001), en nuestro tiempo la sociedad del conocimiento deja paso a la sociedad tecnológica, que a su vez es sucedida por la sociedad del aprendizaje: la persona como conocedora, como fabricante de artefactos destinados a la infraestructura de la sociedad, la persona permanentemente autocrítica, etc., son las características de las imágenes contemporáneas a las que nos enfrentamos. Es aquí donde se enmarca una determinada visión del rendimiento académico.

Sin embargo, el rendimiento académico es un fenómeno complejo que se ve influido por múltiples factores (BARCA et al., 2003), de toda índole. Muchos de estos factores nunca son tenidos en cuenta a lo largo del proceso educativo escolar (NAVARRO, 2003), que es, recordemos, un tiempo obligatorio.

En este marco no deja de ser interesante que las instituciones internacionales se pregunten sobre la preparación del profesorado para hacer frente a estos factores (OECD, 2020). Para Lamas (2015), el estudio del rendimiento académico de los estudiantes es, por su relevancia y complejidad, uno de los temas de mayor controversia en la investigación educativa, a lo cual se le ha dedicado una atención especial en los últimos años (PASSARINI, 2017).

Durante años, una buena parte de los estudios en la educación obligatoria se han centrado en factores relacionados con elementos personales (MELLA; ORTIZ, 1999), 
cosa que aún está en el subconsciente de los miembros de la comunidad educativa y que pone de manifiesto las carencias de los modelos que sustentan estos estudios.

Algunos aspectos estudiados no han sido considerados convenientemente en el debate. Por ejemplo, la importancia del rendimiento previo como claro indicador del éxito posterior (ELVIRA-VALDÉS; PUJOL, 2012), la importancia que debe darse a la orientación y tutoría en las universidades, que en la actualidad posee un modelo de acción muy pobre (TOMÁS-MIQUEL; EXPÓSITO-LANGA; SEMPERE-CASTELLÓ, 2014).

Estos autores consideran que en un modelo universitario donde la exigencia de nuevas metodologías de enseñanza y evaluación ha sido constante, las universidades no han incidido suficientemente en la formación de competencias de organización y hábitos de estudio, así como en un plan de formación en orientación y acción tutorial, aspectos todos los cuales influyen decisivamente en el rendimiento y que comienzan con una ruptura o falta de tradición en la etapa Secundaria y en la transición entre ambas etapas.

Por otro lado, se ha comprobado que el modelo de acceso a la universidad tiene una relación directa con los resultados obtenidos por los estudiantes y también con el abandono (TOMÁS-MIQUEL; EXPÓSITO-LANGA; SEMPERE-CASTELLÓ, 2014), algo que pone de manifiesto, en general, que las competencias a las que el sistema se refiere, presentes tanto en Primaria como en Secundaria, son infravaloradas cuando no despreciadas por formas de acceso monolíticas, inflexibles y poco integradoras.

Este puede ser uno de los puntos más controvertidos del problema (BAUTISTA VALLEJO; LÓPEZ JARA, 2019). Un sistema educativo en donde las competencias son uno de los componentes más importantes, genera una enorme brecha en el momento de la transición, donde determinadas pruebas de acceso a la universidad no consideran la evaluación de las competencias supuestamente adquiridas a lo largo de los años anteriores. Esta puede ser considerada, si no el inicio de la ruptura entre ambos subsistemas educativos, la Secundaria y el universitario, sí la continuación de una relación de desencuentros entre ambas formaciones (DUCOING WATTY; ROJAS MORENO, 2017).

En el ámbito universitario, todos estos enfoques ponen de manifiesto que se ha de incidir en el apoyo y la orientación a los nuevos estudiantes, garantizando que se les presta un mayor apoyo por parte del profesorado, de compañeros de cursos 
superiores, del personal de administración y servicios y de la propia institución universitaria, que ha de dotarle de toda aquella información y consejo que se estime necesario, así como de una atención más personalizada en función de la diversidad de sus expectativas y necesidades, hasta que éstos logren ser autónomos como aprendices (SAGITOVA, 2014). En un sistema que paso a ser, de repente, virtual, todo esto adquiere mucho sentido.

En un análisis de estos elementos, diferentes autores entienden que algunos de los problemas más frecuentes que pueden relacionarse sobre este importante tema Son (ELVIRA-VALDÉS; PUJOL, 2012; TOMÁS-MIQUEL; EXPÓSITO-LANGA; SEMPERECASTELLÓ, 2014; DAZA PÉREZ; TROIANO; ANDREU, 2019; GRAVINA; PRIETO, 2019; NESE et al., 2016; PIERELLA, 2018b):

- $\quad$ Escasa o inexistente orientación previa al acceso a la universidad.

- $\quad$ Desconocimiento de información básica (planes de estudio, etc.).

- $\quad$ Gran diversificación y optatividad del currículum universitario.

- Inadecuada acción tutorial, centrada solo en aspectos académicos.

- $\quad$ Acumulación de desigualdades producidas en elementos previos.

- $\quad$ Efectos negativos que el rendimiento académico ejerce.

- Desenfoque en la formación del profesorado.

\section{Abandono y fracaso educativo}

El acceso de la población joven a la universidad no ha parado de crecer en los últimos años (RAMA; CEVALLOS, 2016). Una diversidad de mecanismos de oferta regional de servicios de educación superior, fundamentalmente públicos pero también privados, son los que han sostenido en los últimos años la expansión de la matrícula y el aumento de la cobertura.

Este movimiento ha hecho posible el ingreso en la universidad de jóvenes cuyos perfiles son cada vez más heterogéneos (DAZA PÉREZ; TROIANO; ANDREU, 2019). Sin embargo, al mismo tiempo ha cuestionado la sensibilidad de la propia institución y los mecanismos de respuesta para esa diversidad. Ante el reto, la propia universidad ha permanecido en muchas ocasiones sin una respuesta clara (BAUTISTA VALLEJO; LÓPEZ JARA, 2019). 
Las reformas de los últimos años han implicado un aumento de oportunidades de matriculación directa en la universidad para muchos jóvenes. La universidad pasó de ser universidad para las elites a universidad masificada (BAUTISTA VALLEJO, 2005), si bien en general, en determinadas regiones como la latinoamericana, el aumento de las demandas colisionó con las desigualdades e inequidades existentes en su funcionamiento, las cuales limitaban su propia masificación (MONTECINOS, 2013).

Este hecho deja claro que a las dificultades de cada subsistema, relacionadas con la organización del currículum, los sistemas de apoyo, etc., hay que sumar otras propias del perfil del alumnado, cada día más heterogéneo y diverso, propio de un sistema en donde aún se da una lucha por la igualdad de oportunidades y la equidad (CONTRERAS CHACÓN; MOLINA YUNCOSA; MÉNDEZ CHACÓN, 2019).

Es decir, durante los últimos años, de forma destacada en el contexto latinoamericano, diversos Estados, organismos e instituciones han puesto su atención no solo en el problema de la calidad de la educación, también en la equidad. Esta es vista cada vez más desde una perspectiva integral, no solo en relación a los mecanismos y procesos que se impulsan, también en relación a la atención a los diversos colectivos a quienes la educación presta sus servicios.

En concreto, esta cuestión debe llevar a explorar cómo el origen social afecta a la entrada de los jóvenes a la universidad desde la vía tradicional (secundaria académica) y desde la vía ampliada (secundaria profesional), algo en lo que se incide desde hace tiempo de forma teórica (BREEN et al., 2009), pero que no termina de hacerse realidad.

Según Daza Pérez, Troiano y Andreu (2019), en países como España, por ejemplo, se evidencian comportamientos diferenciados según el título que poseen los padres y su nivel educativo, siendo en el alumnado de formación profesional los niveles de transición son mucho más bajos.

Torres Navarro (2019), en su análisis sobre los factores que impactan en la deserción estudiantil en las universidades de Latinoamérica, afirma que la mayoría de estos factores se encuentran dentro de tres categorías: económicos, laborales y académicos; este último incluiría, de alguna forma, los factores más personales, como los psicológicos y otros. Dos de ellos englobados dentro de mismo sistema, lo económico-laboral y el otro relativo a la formación-psicología. Visto de otra forma, un 
grupo de factores externos al individuo y uno centrado en la doble visión de los académico, como conjunto de conocimientos que se recibe y como conjunto de elementos que la persona que proyecta procedentes de su propio yo y que las instituciones trabajan hasta aflorar (COSTA MOROSINI et al., 2012).

En este sentido, un contexto económico y laboral pobre, junto con problemas de equidad e igualdad de oportunidades, se presenta como una importante barrera externa que contribuye a la deserción o abandono de la carrera. El conjunto de factores internos se suman y coadyuvan a los anteriores, de forma que en las instituciones educativas, rasgos de personalidad y otros factores psicológicos del alumnado, como el temperamento y la autoestima, podrían estar detrás de la alta tasa de abandono (SMULDERS CHAPARRO, 2018). A esto se suma otros factores como una elección errónea de la carrera y una pobre orientación vocacional durante todo el proceso de transición.

Se trata, pues, de una realidad compleja de resolver a la luz de la naturaleza de las dificultades y, también, de las pocas iniciativas que son implementadas en las instituciones, tanto en los últimos años de Secundaria, como en los primeros universitarios. Esto admite una pluralidad de acciones con las que combatir las tasas de abandono y fracaso educativo.

\section{Discusión y conclusiones}

El peso que se pone a cuestiones como las reformas de los últimos años, el nuevo modelo pedagógico centrado en las competencias, la propia transición Secundaria-universidad, los significados que se le da al rendimiento académico y el manejo del fracaso y abandono en las instituciones, permite trazar unas algunas líneas de trabajo para la reflexión y la intervención. En este sentido, numerosos planteamientos teóricos han recogido planteamientos como la colaboración entre la Secundaria y la universidad, en donde la introducción de la innovación y el trabajo real de las competencias cobran un importante protagonismo en la prevención del fracaso escolar y la formación del profesorado (DOMÍNGUEZ; MEDINA; LÓPEZ, 2018; DUCOING WATTY; ROJAS MORENO, 2017).

Esta necesaria intervención debe darse en el marco de contención de la pandemia por COVID-19, como entienden Wang et al. (2020a). Si bien esta ha 
impactado de forma diversa en los distintos países y continentes, como ha puesto de manifiesto la literatura (SUND, 2020), mantiene medidas tomadas desde los distintos gobiernos, las cuales cambiaron la forma de vivir y trabajar de millones de personas, llevaron de forma casi simultánea, espontánea y, en la mayoría de los casos acelerada, a tomar importantes medidas sociales y, también, educativas (CORNOCK, 2020). Una de ellas fue el teletrabajo, el cual referido a las instituciones educativas consistió en una aceleración en las medidas de virtualización de la enseñanza-aprendizaje.

En este sentido, es importante tener en cuenta que, por ejemplo, en aspectos tan importantes como el de las competencias, lo significativo en torno a las mismas es mantener un discurso abierto, pues las competencias, sean del orden que sean, seguirán siendo comportamientos y capacidades para actuar de maneras deseadas y definidas por otros, como se ha interpretado, con tal de no reducir la autenticidad de la acción humana (BARNETT, 2019).

Una posible solución a este problema permite entender que en la búsqueda de nuevas definiciones de razón que se pretende en estos tiempos (CICARÉ, 2019), el empleo de los niveles más altos de cognición (las metacompetencias y la metacognición) son síntomas de esta nueva búsqueda y, por ende, una manera de superar los posibles problemas relativos a las limitaciones de las propias competencias (BARNETT, 2019). Lo cual implica situar a estos niveles de cognición en el lugar que le corresponde en la etapa secundaria y en la universitaria, hoy poco presentes.

Es decir, el conocimiento metacognitivo desempeña un papel fundamental en la selección y regulación inteligente de estrategias y técnicas de aprendizaje, por lo cual es primordial que el ambiente diseñado permita al alumnado ser consciente de cuáles son las estrategias que mejor le ayuda a aprender, en un conocimiento más profundo de su yo personal y sus circunstancias.

De esta forma, puede concluirse de manera fundamental sobre la conveniencia de desarrollar acciones en los siguientes terrenos:

1) Colaboración entre centros de Secundaria y universitarios, superando la relación existente hoy, que es prácticamente nula. Potenciación de un sistema integrado de orientación en la relación de ambos, con interlocutores válidos y permanentes. 
2) Introducción de la innovación en la relación Secundaria-universidad, superando la situación actual. Es necesario potenciar los factores de éxito académico: atención a estilos y estrategias de aprendizaje del alumnado, aprendizaje personalizado, apoyo a la docencia, calidad de la oferta institucional de servicios de orientación y apoyo, ofrecer un discurso convincente en torno al uso de las TIC, etc.

3) Trabajar las competencias en Secundaria y universidad de una manera seria, teniendo en cuenta el valor profundo de aquellas relacionadas con la capacidad de aprender, tener iniciativa, autonomía, emprender, etc. Las competencias como lenguaje, contenido y expresión pedagógica debe continuar en la universidad. Es necesario superar, de esta forma, el valor del contenido para ir más allá, y entender y poner en marcha el sentido profundo de la competencia.

4) Para todo ello es necesario un programa de formación docente más ambicioso, que fortalezca no solo contenidos sino, también, las metodologías docentes que se presentan, como proceso, igualmente como contenidos que son aprehendidos por parte del alumnado. En ello, los enfoques centrados en el alumnado y el aprendizaje, en el marco de los nuevos conocimientos sobre la cognición como proceso son fundamentales.

\section{Referencias}

ÁLVAREZ COLÍN, Luis. La educación basada en competencias: implicaciones, retos y perspectivas. Didac, México, n. 36, p. 26-33, 2000.

ARGUDÍN, Yolanda. La educación superior para el siglo XXI. Didac, México, n. 36, p. 16$25,2000$.

BANCO MUNDIAL. Graduarse: solo la mitad lo logra en América Latina. Washington: Banco Mundial, 2017.

BARCA, Alfonso. et al. Enfoques de aprendizaje, rendimiento académico y género en alumnos de educación secundaria (ESO): un análisis diferencial. Psicología, Educação e Cultura, Carvalhos, n. 2, p. 25-43, 2003.

BARDIN, Laurence. Análisis de contenido. Madrid: Akal, 1996.

BARNETT, Ronald. Los límites de la competencia. Barcelona: Gedisa, 2001. 
BARNETT, Ronald. The thoughtful university: a feasible utopia. Beijing International Review of Education, Leiden, v. 1, n. 1, p. 55-72, 2019.

BAUTISTA VALLEJO, José Manuel. Universidad y espacio europeo en la encrucijada de la calidad. Huelva: Hergué, 2005.

BAUTISTA VALLEJO, José Manuel; LÓPEZ JARA, Norma Raquel. Análisis crítico del modelo basado en competencias en la Universidad. Academo, Asunción, v. 6, n. 1, p. 71-80, 2019.

BAUTISTA-VALLEJO, José Manuel; ESPIGARES-PINAZO, Manuel J.; HERNÁNDEZCARRERA, Rafael M. El EEES en la Universidad española como innovación y el papel del alumnado veinte años después. In: HINOJO-LUCENA, F. J.; AZNAR-DÍAZ, I.; CÁCERES, M. P. (ed.). Avances en recursos TIC e innovación educativa. Madrid: Dykinson, 2019. p. 139-151.

BOUZA MORA, D. Modificación de las actividades académicas durante la situación de emergencia por COVID19 en la Universidad Nacional. San José, Costa Rica: Universidad Nacional, 2020.

BREEN, Richard et al. Nonpersistent Inequality in Educational Attainment: Evidence from Eight European Countries. American Journal of Sociology, Chicago, n. 114, p. 1475-1521, 2009.

CASTILLO, Juan José; MORÉ, Paloma. Universidad precaria, universidad sin futuro. Madrid: Fuhem Editorial, 2018.

CICARÉ, Adriana C. ¿Hacia dónde van las políticas públicas en educación? una mirada internacional y mercosureña. Revista de la Secretaría del Tribunal Permanente de Revisión, Asunción, v. 7, n. 14, p. 295-312, 2019. Disponível em: https://doi.org/10.16890/rstpr.a7.n14.p295. Acesso em: 8 nov. 2020.

CONTRERAS CHACÓN, Azael Eduardo; MOLINA YUNCOSA, Nuby Lisbeth; MÉNDEZ CHACÓN, Gusmary del Carmen. Observatorio regional de calidad y equidad de la educación superior en latinoamerica (ORACLE). Educere, Umuarama, v. 23, n. 76, p. 687-697, 2019.

CORNOCK, Matt. Scaling up online learning during the coronavirus (Covid-19) pandemic. 2020. Disponível em: https://mattcornock.co.uk/technology-enhancedlearning/scaling-up-online-learning-during-the-coronavirus-covid-19-pandemic. Acesso em: 7 nov. 2020.

COSTA MOROSINI, Marília Costa et al. A evasão na Educação Superior no Brasil: uma análise da produção de conhecimento nos periódicos Qualis entre 2000-2011. In: ANDOAÍN, G.; ARRIAGA, J. Memoria primera Conferencia Latinoamericana sobre el abandono en la Educación Superior, I CLABES. Managua: Universidad Nacional Autónoma de Nicaragua, 2012. p. 65-73. 
DAZA PÉREZ, Lidia Daza; TROIANO, Helena; ANDREU, Marina Elias. La transición a la universidad desde el bachillerato y desde el CFGS. La importancia de los factores socioeconómicos. Papers: Revista de Sociología, Barcelona, v. 1, n. 1, p. 1-21, 2019.

DELORS, Jacques. La educación encierra un tesoro: Informe a la UNESCO de la Comisión Internacional sobre la Educación para el Siglo XXI. Madrid: Santillana: UNESCO, 1996.

DOMÍNGUEZ, María Concepción; MEDINA, Antonio; LÓPEZ, Ernesto. Desarrollo de competencias en el primer curso de universidad: estudio de caso. Publicaciones, Melilla, v. 48, n. 1, p. 39-62, 2018.

DUCOING WATTY, Patricia; ROJAS MORENO, Ileana. La educación secundaria en el contexto latinoamericano: consideraciones a partir del vínculo política educativacurrículum. Revista mexicana de investigación educativa, Ciudad de México, v. 22, n. 72, p. 32-56, 2017.

DUQUE, Alba Patricia Guzmán; CONTRERAS, Diana Oliveros; GARCÍA, Edgar Mauricio Mendoza. Las competencias científicas a partir de la gestión del conocimiento en Instituciones de Educación Superior. Signos: Investigación en sistemas de gestión, Bogotá, v. 11, n. 2, p. 21-40, 2019.

ELVIRA-VALDÉS, María Antonieta; PUJOL, Lydia. Autorregulación y rendimiento académico en la transición secundaria-universidad. Revista Latinoamericana de Ciencias Sociales, Niñez y Juventud, Santiago de Chile, v. 10, n. 1, p. 367-378, 2012.

GIL-GALVÁN, Rosario. El absentismo en la enseñanza universitaria desde la perspectiva de los estudiantes. Educação e Pesquisa, São Paulo, n. 45, p. e188660, 2019.

GONZÁLEZ, Julia; WAGENAAR, Robert. Tuning educational structures in Europe: informe final. Bilbao: Universidad de Deusto, 2003.

GRAVINA, Virginia; PRIETO, Víctor. La brecha secundaria-universidad: diagnóstico de los estudiantes de Agronomía en el curso inicial de matemáticas. Intercambios: Dilemas y Transiciones de la Educación Superior, Montevideo, v. 6, n. 1, p. 34-42, 2019.

HERNÁNDEZ CARRERA, Rafael Manuel. La investigación cualitativa a través de entrevistas: su análisis mediante la teoría fundamentada. Cuestiones Pedagógicas, Sevilla, n. 23, p. 187-210, 2014.

HEYWOOD, Lindsay; GONCZI, Andrew; HAGER, Paul. Guide to development of competence-based standards for professions. Camberra: Australian Government Publishing Service, 1993.

ISERN, Icart M. T. Y; CANELA-SOLER, J. El artículo de revisión. Enfermería Clínica, Barcelona, v. 4, n. 4, p. 180-184, 1994. 
KAUARK, Fabiana da Silva; MANHÃES, Fernanda Castro; MEDEIROS, Carlos Henrique. Metodologia da pesquisa: um guia prático. Itabuna: Via Litterarum, 2010.

KROTSCH, Pedro. La universidad en el proceso de integración regional: el caso del MERCOSUR. Perfiles educativos, México, v. 19, n. 77, p. 1-26, 1997.

LAMAS, Héctor A. Sobre el rendimiento escolar. Propósitos y Representaciones, Lima, v. 3, n. 1, p. 313-386, 2015.

MARÍN, Francisco Guzmán. El concepto de competencias. Contexto Odontológico, México, v. 5, n. 10, ago. 2018.

MEIRIEU, Philippe; MONTENEGRO GÓMEZ, Paula Alexandra. Riquezas y límites del enfoque por "competencias" del ejercicio de la profesión docente hoy. Pedagogía y Saberes, Bogotá, n. 50, p. 97-108, 2019.

MELLA, Orlando; ORTIZ, Iván. Rendimiento escolar: influencias diferenciales de factores externos e internos. Revista Latinoamericana de Estudios Educativos, México, v. 29, n. 1, p. 69-92, 1999.

MONTECINOS, Egon. Los actuales desafíos regionales en Chile: ¿nueva regionalización o más descentralización? Los Lagos: Universidad de Los Lagos: Centro de Estudios del Desarrollo Regional y Local, 2013.

MORA, José Ginés. Universidades: mitos, modas y tendencias. Revista Iberoamericana de Educación Superior, Madrid, v. 9, n. 24, p. 3-16, 2018.

NAVARRO, Ruben Edel. Factores asociados al rendimiento académico. Revista Iberoamericana de Educación, Madrid, v. 33, n. 1, p. 1-20, 2003.

NESE, Rhonda et al. Predicting abandonment of school-wide positive behavioral interventions and supports. Behavioral Disorders, Illinois, v. 42, n. 1, p. 261-270, 2016.

OECD - ORGANISATION FOR ECONOMIC CO-OPERATION AND DEVELOPMENT. How prepared are teachers and schools to face the changes to learning caused by the coronavirus pandemic?. Paris: OECD Publishing, 2020. (Teaching in Focus, n. 32).

PASSARINI, José. Trayectorias, egreso y acreditación de carreras en el Mercosur. InterCambios: Dilemas y Transiciones de la Educación Superior, Montivideo, v. 4, n. 2, p. 36-49, 2017.

PEREIRA, Elisabete Monteiro Aguiar; PASSOS, Rogério Duarte Fernandes. 0 espaço europeu de ensino superior e cidadania europeia. Revista Internacional de Educação Superior, Campinas, v. 4, n. 1, p. 175-196, 2018. Disponível em: https://doi.org/10.22348/riesup.v4i1.8651136. Acesso em: 10 out. 2020.

PERRY, John; LUNDIE, David; GOLDER, Gill. Metacognition in schools: what does the literature suggest about the effectiveness of teaching metacognition in schools? Educational Review, Edinburgh, v. 71, n. 4, p. 483-500, 2018. 
PIERELLA, María Paula. A 100 años de la reforma: desafíos de la enseñanza en los primeros años de la universidad. Trayectorias Universitarias, Buenos Aires, v. 4, n. 6, p. 16-22, 2018a.

PIERELLA, María Paula. El primer año de universidad desde la perspectiva de los profesores. Políticas de recepción, enseñanza y curriculum. Espacios en Blanco. Revista de Educación, Santiago, n. 28, p. 161-182, 2018b.

PRIETO, Leonor (coord.). La enseñanza universitaria centrada en el aprendizaje. Barcelona: Octaedro: ICE UB, 2008.

RAMA, Claudio; CEVALLOS, Marcelo. Nuevas dinámicas de la regionalización universitaria en América Latina. Magis, Revista Internacional de Investigación En Educación, Bogotá, v. 8, n. 17, p. 99-134, 2016.

RODRÍGUEZ ESPINAR, Sebastián. Nuevos retos y enfoques en la formación del profesorado universitario. Revista de educación, v. 331, p. 67-99, 2003.

RODRÍGUEZ, A.; DAPÍA, M. D.; LÓPEZ-CASTEDO, A. Ansiedad ante los exámenes en alumnos de Educación Secundaria Obligatoria. Revista de Estudios e Investigación en Psicología y Educación, v. 1, n. 2, p. 132-140, 2014.

ROLÓN, Violeta. Enfoques curriculares en la Educación Superior. Academo, Asuncón, v. 3, n. 1, 2016.

SAFITRI, Dian et al. Students' cognitive achievement, critical thinking skills, and metacognitive awareness in problem based learning. European Journal of Education Studies, Bucharest, v. 5, n. 4, p. 248-258, 2018.

SAGITOVA, R. R. Training students to be autonomous learners. International Journal of Humanities Education, [s. l.], v. 12, n. 1, p. 27-34, 2014.

SMULDERS CHAPARRO, Marta Elena. Factores que influyen en la deserción de los Estudiantes Universitarios. Academo, Asunción, v. 5, n. 2, p. 127-132, 2018.

SOARES, Adriana Benevides et al. Expectativas acadêmicas de estudantes nos primeiros anos do Ensino Superior. Arquivos Brasileiros de Psicologia, Rio de Janeiro, v. 70, n. 1, p. 206-223, 2018.

SOUSA, Sumária; SANTOS JUNIOR, Antônio Carlos Pereira. Google sala de aula como ambiente virtual de aprendizagem no ensino superior híbrido: uma revisão da literatura. EAD em Foco, Rio de Janeiro, v. 9, n. 1, p. e768, 2019.

SUND, Kristian J. Suggestions for moving teaching rapidly online in the face of the corona crisis. Roskilde: Roskilde University, 2020.

TOMÁS-MIQUEL, José-Vicente; EXPÓSITO-LANGA, Manuel; SEMPERE-CASTELLÓ, Saúl. Determinantes del rendimiento académico en los estudiantes de grado: un estudio en administración y dirección de empresas. Revista de Investigación Educativa, Salamanca, v. 32, n. 2, p. 379-392, 2014. 
TORI, Romero. Educação sem distância: as tecnologias interativas na redução de distâncias em ensino e aprendizagem. 2. ed. São Paulo: Artesanato Educacional, 2017.

TORRES NAVARRO, Luis Abel. Factores que impactan en la deserción estudiantil en las universidades de Latinoamérica del año 2009 al 2019: una revisión de la literatura científica. Lima: Universidad Privada del Norte, 2019.

TORRES-LÓPEZ, Surayne et al. Evaluación de competencias laborales a partir de evidencias. Ingeniería Industrial, La Habana, v. 39, n. 2, p. 124-134, 2018.

WANG, Guanghai et al. Mitigate the effects of home confinement on children during the COVID-19 outbreak. The Lancet, London, p. 1-2, 4 mar. 2020a.

WANG, Chuanyi et al. Risk management of COVID-19 by Universities in China. Journal of Risk and Financial Management, Basel, v. 13, n. 2, p. 1-6, 2020b.

YORKE, Mantz. Leaving early: Undergraduate non-completion in higher education. Psychology Press, London: 1999.

Recebido em: 16 abril 2021

Aceite em: 15 outubro 2021 